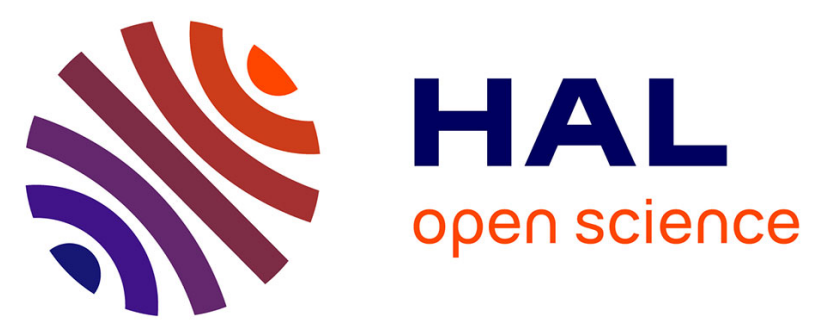

\title{
Photoionization and dissociative photoionization of propynal in the gas phase: theory and experiment
} Imene Derbali, Helgi Rafn Hrodmarsson, Zied Gouid, Martin Schwell, Marie-Claire Gazeau, Jean-Claude Guillemin, Majdi Hochlaf, Mohammad Esmail Alikhani, Emilie-Laure Zins

\section{To cite this version:}

Imene Derbali, Helgi Rafn Hrodmarsson, Zied Gouid, Martin Schwell, Marie-Claire Gazeau, et al.. Photoionization and dissociative photoionization of propynal in the gas phase: theory and experiment. Physical Chemistry Chemical Physics, 2019, 21 (26), pp.14053-14062. 10.1039/C8CP06751A . hal02095692

HAL Id: hal-02095692

https://hal-univ-rennes1.archives-ouvertes.fr/hal-02095692

Submitted on 10 Apr 2019

HAL is a multi-disciplinary open access archive for the deposit and dissemination of scientific research documents, whether they are published or not. The documents may come from teaching and research institutions in France or abroad, or from public or private research centers.
L'archive ouverte pluridisciplinaire HAL, est destinée au dépôt et à la diffusion de documents scientifiques de niveau recherche, publiés ou non, émanant des établissements d'enseignement et de recherche français ou étrangers, des laboratoires publics ou privés. 


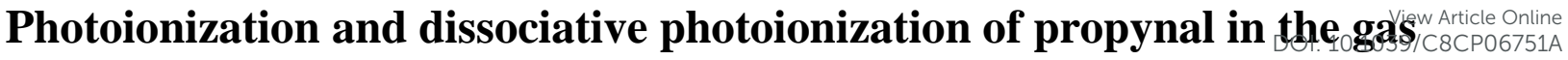 phase: Theory and experiment}

Imene Derbali, ${ }^{1}$ Helgi Rafn Hrodmarsson, ${ }^{2}$ Zied Gouid, ${ }^{3}$ Martin Schwell, ${ }^{4,}$ Marie-Claire Gazeau, ${ }^{4}$ Jean-Claude Guillemin, ${ }^{5}$ Majdi Hochlaf, ${ }^{3 *}$ Mohammad Esmaïl Alikhani, ${ }^{1}$ Emilie-Laure Zins ${ }^{1 *}$

${ }^{1}$ MONARIS UMR 8233 CNRS, Sorbonne Université, 4 place Jussieu, 75252 Paris Cedex 5, France.

${ }^{2}$ Synchrotron SOLEIL, L'Orme des Merisiers, 91192 Saint Aubin, Gif-sur-Yvette, France

${ }^{3}$ Université Paris-Est, Laboratoire Modélisation et Simulation Multi Echelle, MSME UMR 8208 CNRS, 5 bd Descartes, 77454 Marne-la-Vallée, France.

${ }^{4}$ LISA UMR CNRS 7583, Université Paris Est Créteil and Université Paris Diderot, Institut Pierre Simon Laplace, 61 Avenue du Général de Gaulle, 94010 Créteil, France

${ }^{5}$ Univ. Rennes, Ecole Nationale Supérieure de Chimie de Rennes, CNRS, ISCR-UMR6226, F-35000 Rennes, France

* Corresponding authors: Emails: martin.schwell@lisa.u-pec.fr; hochlaf@univ-mlv.fr; emilielaure.zins@sorbonne-universite.fr 


\section{ABSTRACT}

Propynal (HCCCHO) is a complex organic compound (COM) of astrochemical and astrobiological interest. We present a combined theoretical and experimental investigation on the single photon ionization of gas-phase propynal, in the 10 to $15.75 \mathrm{eV}$ energy range. Fragmentation pathways of the resulting cation were investigated both theoretically and experimentally. The adiabatic ionization energy (AIE) has been measured to be $\mathrm{AIE}_{\exp }=10.715 \pm 0.005 \mathrm{eV}$ using tunable VUV synchrotron radiation coupled with a double imaging photoelectron photoion coincidence ( $\left.{ }^{2} \mathrm{PEPICO}\right)$ spectrometer. In the energy range under study, three fragments formed by dissociative photoionization were identified experimentally: $\mathrm{HC}_{3} \mathrm{O}^{+}, \mathrm{HCO}^{+}$and $\mathrm{C}_{2} \mathrm{H}_{2}^{+}$, and their respective appearance energies (AE) were found to be $\mathrm{AE}=11.26 \pm 0.03,13.4 \pm 0.3$ and $11.15 \pm 0.03 \mathrm{eV}$, respectively. Using explicitly correlated coupled cluster calculations and after inclusion of the zero point vibrational energy, core-valence and scalar relativistic effects, the $\mathrm{AIE}$ is calculated to be $\mathrm{AIE}_{\text {calc }}=10.717 \mathrm{eV}$, in excellent agreement with the experimental finding. The appearance energies of the fragments were calculated using a similar methodological approach. To further interpret the observed vibrational structure, anharmonic frequencies were calculated for the fundamental electronic state of the propynal cation. Moreover, MRCI calculations were carried out to understand the population of excited states of the cationic species. This combined experimental and theoretical study will help to understand the presence and chemical evolution of propynal in the external parts of interstellar clouds where it has been observed. 


\section{Introduction}

With the latest telescopes such as the Atacama Large Millimeter Array (ALMA), the Northern Extended Millimeter Array (NOEMA), the Green Bank Telescope (GBT) and others, we are witnessing an unprecedented acceleration of our knowledge of the chemistry of the interstellar medium (ISM). More than 205 molecules, up to 13 atoms large, are observed today. In order to fully interpret scientifically the numerous radio telescopes measurements, supporting laboratory studies must be performed. They should cover a wide range of chemical reactions and processes that may occur under interstellar conditions. In this context, databases and chemical networks dedicated to the study of interstellar physical chemistry, such as $\mathrm{KIDA}^{1}$, play an important role since they deepen our understanding of the chemical processes that occur in the ISM.

Propynal $\left(\mathrm{H}_{2} \mathrm{C}_{3} \mathrm{O}\right)$, the object of our study, is a small organic molecule containing three of the four atoms essential to life as we know it on Earth. It was identified in the ISM, as early as 1988 in the TMC-1 molecular cloud. ${ }^{2}$ Shortly after, Adams et al. ${ }^{3}$ have proposed that the $\mathrm{H}_{2} \mathrm{C}_{3} \mathrm{O}^{+}$ion could be formed by radiative association reactions involving smaller hydrocarbon ions and carbon monoxide. Later, propynal was also detected in Sagittarius B2(N). ${ }^{4,5}$ The most recent radioastronomical study led to the identification of propynal in different molecular clouds: TMC-1, B1-b, L483, Lupus-1A, L1495B, L1521F and Serpens South 1a. ${ }^{6}$ More than a decade of laboratory astrochemistry studies on interstellar ice analogues permitted to propose formation mechanisms for many molecules in the ISM. Indeed, the formation of propynal, alongside with its isomer cyclopropenone, has been observed in the laboratory upon irradiation of $\mathrm{CO} / \mathrm{C}_{2} \mathrm{H}_{2}$ ices with high energy electrons at $10 \mathrm{~K} .{ }^{7}$ On the other hand, gas phase reactions such as elementary photoreactions and bimolecular reactions can involve molecules after they have desorbed from ices or grains. The importance of chemical processes occurring directly in the gas phase has recently been highlighted by Loison et al. ${ }^{6}$ With the help of the Nautilus chemical code ${ }^{8}$ in combination with observations in several interstellar objects, these authors concluded that the interstellar abundances of the three members of the $\mathrm{H}_{2} \mathrm{C}_{3} \mathrm{O}$ isomer family (propynal, cyclopropenone and propadienone) are controlled by gas phase kinetics. We finally note that the presence of propynal in comet P/Halley has also been suggested. ${ }^{9}$ According to this study, its gas phase photolysis yields $\mathrm{C}_{3}$, $\mathrm{CO}$ and $\mathrm{C}_{2} \mathrm{H}_{2}$ all observed in-situ by the VEGA space probe during its flyby in 1986.

Following the first experimental photoelectron spectrum of Carlier et al. in $1979,{ }^{10}$ several theoretical studies have focused on the reactivity of neutral propynal, its photoionization and photodissociation. ${ }^{11,12,13}$ In 1991, Opitz carried out a multiphoton ionization study of the fragmentation of propynal using laser radiation at $\lambda=193$ and $248 \mathrm{~nm} .{ }^{14}$ To our knowledge, this is the only experimental study of the photoionization of propynal. Opitz observed the formation of $\mathrm{HC}_{3} \mathrm{O}^{+}(\mathrm{m} / \mathrm{z}=$ 53), $\mathrm{CO}^{+}(\mathrm{m} / z=28), \mathrm{C}_{2} \mathrm{H}_{2}{ }^{+}(\mathrm{m} / z=26)$ and $\mathrm{C}_{2}{ }^{+}(\mathrm{m} / z=24)$ cationic fragments. In 2009 , a theoretical study was published by Holmes et al. ${ }^{15}$ It focused on the formation and ionization energies of a representative 
panel of isomers of the empirical formulas $\mathrm{C}_{3} \mathrm{H}_{2} \mathrm{O}$ and $\mathrm{C}_{4} \mathrm{H}_{4} \mathrm{O}$. Fully relevant for astrochemistryyiethisicle Online study allows the prediction of the ionization thresholds of neutral isomers potentially present in the ISM.

Propynal formed in interstellar clouds or in the vicinity of comets can easily undergo gas phase photoionization. By subsequent dissociation of the parent cation, radical cations of high reactivity can be produced and in turn, influencing the ongoing chemistry. The study of the photoionization of astrochemical molecules in the VUV spectral domain is particularly relevant, since the edges of molecular clouds are subject to intense VUV radiation. These photons have energies between 6.0 and $13.6 \mathrm{eV}$. More energetic photons are absorbed by atomic hydrogen. ${ }^{16}$ Energetic particles and electrons can also induce dissociative ionization processes. It is therefore highly relevant to study the photoionization of propynal from its adiabatic ionization energy (AIE) up to $15.75 \mathrm{eV}$ in order to provide accurate data for realistic modeling of the chemistry of interstellar clouds, and in particular the processes of photoionization and photodissociation as well as the identification of the fragments formed as a function of energy and of their appearance energies (AEs).

\section{Methods}

\subsection{Experimental methods}

The experiments were carried out at the undulator-based DESIRS beamline of the French synchrotron facility SOLEIL ${ }^{17}$ in connection with its $6.65 \mathrm{~m}$ normal incidence monochromator and the double imaging photoelectron photoion coincidence (i²PEPICO) spectrometer DELICIOUS3. ${ }^{18}$ This spectrometer allows for full momentum detection of the ions and velocity map imaging (VMI) of the electrons at the same time, but in exactly opposite directions. Ions and electrons were detected in coincidence and their collection is perpendicular to i) the molecular beam inlet and ii) the VUV light propagation direction (all $90^{\circ}$ setup). More details on this set-up and the experimental operating conditions can be found in ref. ${ }^{18}$ For our measurements, we used the 200 lines/mm grating of the monochromator with entrance/exit slit widths of typically $100 \mu \mathrm{m} / 100 \mu \mathrm{m}$ yielding a spectral resolution (photons) of $0.72 \AA$ (about $6 \mathrm{meV}$ at $10 \mathrm{eV}$ ). (T)PEPICO spectra were normalized by the photon flux measured by a photodiode (AXUV, IRD) placed after the photoionization region. For better spectral purity of the beamline, we used a gas filter that is filled with 0.25 mbar of Ar. This allows for effective suppression of either higher energy stray light of the electron storage ring or higher order radiation from the undulator. The presence of Ar absorption lines in the spectrum arising from this filter furthermore allows for calibration of the energy scale to an absolute accuracy of about $1 \mathrm{meV}$.

Propynal has been synthesized as reported in ref. ${ }^{19}$ A degassed sample can be kept for months at $\mathrm{T}=-30^{\circ} \mathrm{C}$ in a cell under vacuum. The compound is sufficiently stable at room temperature for measurements with DELICIOUS3. 
The main aim of the present computational study is to provide an interpretation of the experimental results. These theoretical investigations include geometry optimizations, the determination of the ionization energy as well as the vibrational frequencies of neutral and ionic propynal. The fragmentations of the propynal cation were also theoretically investigated. All the calculations were carried out with the Gaussian09-Revision $\mathrm{E}^{20}$ and MOLPRO ${ }^{21}$ suites of programs. All calculations were carried out in the $C_{l}$ point group. Theoretical calculations on the electronic excited states of the propynal cation were also carried out, after a molecular orbital (MO) characterization.

Recently, it has been shown that AIEs may be obtained using the explicitly correlated coupled clusters ((R)CCSD (T)-F12) method after inclusion of core-valence (CV), scalar relativistic (SR) and zero point energy (ZPE) corrections. ${ }^{22,23}$ Geometry optimizations were first done using the (R)CCSD(T)F12 method ${ }^{24,25,26,27}$ where the atoms were described using the aug-cc-pVTZ basis sets ${ }^{28}$. The CV effects are deduced as the difference between electronic energies with only valence electrons correlated and that with both core and valence electrons correlated at the (R)CCSD(T)/cc-pwCVTZ level ${ }^{29}$ of theory. The core electrons are the 1s electrons of carbon and oxygen atoms. As an example, the calculation of the $\triangle \mathrm{CV}$ contribution for the formation of the $\mathrm{A}^{+}+\mathrm{B}^{\circ}$ fragments is given in Eq.1:

$$
\Delta C V=\left[E_{F u l l}^{A+}+E_{F u l l}^{B^{\circ}}-E_{F u l l}^{\text {Neutral }}\right]-\left[E_{F C}^{A+}+E_{F C}^{B^{\circ}}-E_{F C}^{\text {Neutral }}\right] \text { (Eq. 1) }
$$

The SR energetic contributions are calculated as the difference between electronic energies at the (R)CCSD(T)/cc-pVTZ ${ }^{30}$ level without using the spin-free, one electron Douglas-Kroll-Hess (DKH) $)^{31,32}$ Hamiltonian and at the (R)CCSD(T)/cc-pVTZ-DK ${ }^{33}$ level with the DKH Hamiltonian. Eq.2 gives an example of the calculation of the $\Delta \mathrm{SR}$ correction for the formation of the $\mathrm{A}^{+}+\mathrm{B}^{\circ}$ fragments:

$$
\begin{aligned}
\Delta S R= & {\left[E_{(\mathrm{R}) \operatorname{CCSD}(\mathrm{T})+D K H}^{A+}+E_{(\mathrm{R}) \mathrm{CCSD}(\mathrm{T})+D K H}^{B^{\circ}}-E_{(\mathrm{R}) \operatorname{CCSD}(\mathrm{T})+D K H}^{\text {Neutral }}\right] \quad \text { (Eq. 2) } } \\
& -\left[E_{(\mathrm{R}) \operatorname{CCSD}(\mathrm{T})}^{A+}+E_{(\mathrm{R}) \operatorname{CCSD}(\mathrm{T})}^{B^{\circ}}-E_{(\mathrm{R}) \operatorname{CCSD}(\mathrm{T})}^{\text {Neutal }}\right]
\end{aligned}
$$

The zero-point variational energy correction (ZPE) and anharmonic frequencies were obtained at the PBE0 level with aug-cc-pVTZ basis. ${ }^{28,30,34}$

Afterwards, theoretical AIEs and AEs were evaluated using the following formula (Eq. 3):

$$
E=\Delta E_{(R) C C S D(T)-F 12}+\Delta C V+\triangle S R+\triangle Z P E \quad \text { (Eq. 3) }
$$

where $E$ stands for AIE or AE and $\Delta E_{(R) C C S D(T)-F 12}$ corresponds to the energy difference between the neutral and corresponding cationic species as evaluated at their equilibrium geometries using (R)CCSD(T)-12 approach.

The excited electronic states of the propynal cation have been also investigated using MOLPRO suite of programs, in the $C_{s}$ point group. We have used the state-averaged complete active space selfconsistent field $\mathrm{CASSCF}^{35,36}$ method followed by the internally contracted multireference configuration

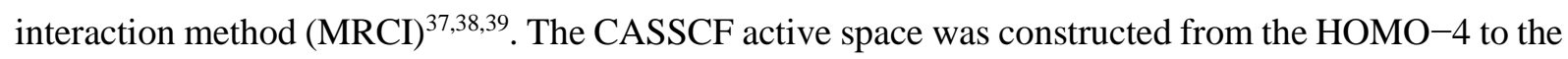


LUMO+3 molecular orbitals, where we requested four ${ }^{2} \mathrm{~A}^{\prime}$ and four ${ }^{2} \mathrm{~A}^{\prime \prime}$ states. At MRCliewallicle Online configurations with coefficients larger than 0.05 in the CI expansion of the CASSCF wavefunctions were used as a reference. This results into more $12.5 \times 10^{6}$ uncontracted configuration state functions to be treated per $\mathrm{C}_{\mathrm{s}}$ symmetry. Again, four ${ }^{2} \mathrm{~A}^{\prime}$ and four ${ }^{2} \mathrm{~A}^{\prime}$ states were requested. We also performed electronic excited states of some fragments when needed using similar methodology.

\section{Results and Discussion}

We present a combined theoretical and experimental investigation on the single photon ionization of the propynal molecule in the gas-phase, in the 10 to $15.75 \mathrm{eV}$ energy range. Calculations were carried out in order to assign the observed ionization and fragmentation thresholds. To this end, a calibrated method of theory was applied. Further calculations were performed with the aim to elucidate the structure of fragment ions experimentally observed.

\subsection{The photoionization mass spectrum}

The time-of-flight mass spectrum obtained with a photon energy of $h v=15 \mathrm{eV}$ is presented in Figure 1. Four ions were identified: the parent ion $\mathrm{C}_{3} \mathrm{H}_{2} \mathrm{O}^{+}(\mathrm{m} / z$ 54), the fragment ion corresponding to the loss of atomic hydrogen $\mathrm{HC}_{3} \mathrm{O}^{+}(\mathrm{m} / \mathrm{z} 53), \mathrm{HCO}^{+}(\mathrm{m} / \mathrm{z} 29)$, and $\mathrm{C}_{2} \mathrm{H}_{2}{ }^{+}(\mathrm{m} / \mathrm{z} 26)$ and their ${ }^{13} \mathrm{C}$ isotopologues. As can be seen from Fig. $1, \mathrm{HC}_{3} \mathrm{O}^{+}$and $\mathrm{C}_{2} \mathrm{H}_{2}{ }^{+}$are very intense ions and can be considered as the two main cationic fragments formed by dissociative photoionization of propynal in the energy regime under study here. $\mathrm{HCO}^{+}$, on the contrary, is very low in intensity and can only be seen in the mass spectrum when zooming in the baseline (not shown). We note the absence of the $\mathrm{CO}^{+}$ion $(\mathrm{m} / \mathrm{z}, 28)$ that was observed earlier by Opitz in the multi-photon regime, at $\lambda=193 \mathrm{~nm} .{ }^{14}$ Note that in ref. ${ }^{14} \mathrm{CO}^{+}$ is only seen at photoexcitation at $\lambda=193 \mathrm{~nm}$ and laser intensities above $\mathrm{P}=5 \times 10^{7} \mathrm{~W} \cdot \mathrm{cm}^{-2}$. At such intensities, population of higher excited states well above $15 \mathrm{eV}$ cannot be avoided. Thus, we suggest that in their work, $\mathrm{CO}^{+}$is formed by photodissociation of higher excited states of propynal, well above the photon energy regime of our study.

\subsection{Ionization and fragmentation thresholds}

All experimental and theoretical results concerning ionization and fragmentation thresholds are summarized in Table 1. The experimental AIE of propynal is determined from the TPEPICO spectrum of the ion ground state shown in Figure 2. It is measured to be $\mathrm{AIE}_{\exp }=10.715 \pm 0.005 \mathrm{eV}$. This value corresponds to the median of the $0_{0}^{0}$ transition from the neutral ground state to the fundamental state of the cation, indicated by a vertical dotted line in Fig. 2. Our AIE measurement is in good accordance with the electron impact (EI) ionization measurement of ref..$^{14}(10.62 \pm 0.15 \mathrm{eV})$, but more accurate by a factor of 30. It is also in good accordance with values deduced from the early photoelectron spectroscopic measurements $\left(10.70 \pm 0.01 \mathrm{eV}^{10}\right.$ and $\left.10.8 \mathrm{eV}^{40}\right)$. In the work presented in ref. ${ }^{40}$ no error bars are given 
for the AIE but it is probably less precise than the AIE from ref. ${ }^{10} \mathrm{~A}$ most recent theorreticalcle Online determination, at the Gaussian-4 (G4) level of theory, is given in ref. ${ }^{41}$ to be $\mathrm{AIE}_{\text {calc }}=10.71 \mathrm{eV}$. The observed vibronic progression in the ground state TPEPICO spectrum shown in Fig. 2 will be analysed in paragraph 3.4 .

In the present work, the AIE value is calculated $\mathrm{AIE}_{\text {calc }}=10.717 \mathrm{eV}$ at the $(\mathrm{R}) \mathrm{CCSD}(\mathrm{T})-\mathrm{F} 12$ level, including $\Delta \mathrm{CV}(+0,00037 \mathrm{eV}), \Delta \mathrm{SR}(-0.0000055 \mathrm{eV})$ and $\Delta \mathrm{ZPE}(-0.001854 \mathrm{eV})$ corrections. Our value is in excellent agreement with our experimental finding since it falls into the precision interval $( \pm 0.005 \mathrm{eV})$ of the experimental AIE. In addition, our calculated AIE is more accurate than the most recent calculation from ref. ${ }^{41}$ This is in line with recent determinations of AIEs of medium-sized molecular systems using similar experimental and theoretical approaches. ${ }^{22,23,42,43,44,45}$

In order to investigate the dissociative ionization processes we present in Figure 3 the threshold of formation regions of the three observed fragment cations in the experiments: $m / z 53$ (Fig. 3a), $m / z 26$ (Fig. 3b) and $m / z 29$ (Fig. 3c). In order to determine the respective AEs, we insert a blue line indicating the noise level (false coincidences) of the baseline. The experimental AEs are set at the energy where the mass signal exceeds the noise level. The values determined in this manner are listed in Table 1 . The case of $\mathrm{m} / \mathrm{z} 53$ is special since it shows that the baseline is rising slightly with energy. This is because the $\mathrm{m} / \mathrm{z} 53$ signal is contaminated, to some minor extent, by the neighboring strong $\mathrm{m} / \mathrm{z} 54$ signal of the parent ion. The latter is rising in the energy regime of Fig. 3a. We consider that this small contamination is insignificant for the AE determination of $m / z$ 53. In Figs. 3a-c we have added black horizontal bars in the threshold region in order to indicate the error that is estimated from this graphical AE determination $( \pm 0.03 \mathrm{eV}$ for $\mathrm{m} / \mathrm{z} 53$ and $\mathrm{m} / \mathrm{z}, 26$ ). The intensity of the ion signal $\mathrm{m} / \mathrm{z} 29$ is much lower than compared to the principal fragment ions $m / z 53,26$. Furthermore, the $m / z, 29$ ion formation onset is very smooth. This is why the $\mathrm{AE}$ of this cation can only be determined with much less precision $( \pm 0.3 \mathrm{eV})$.

In order to understand the dissociative photoionization in more detail, different fragmentation pathways were considered in the quantum-chemical calculations. They are presented in Figure 4. The respective calculated AEs are summarized in Table 1 . For $m / z 53$, corresponding to the $\mathrm{H}$ loss reaction, two isomeric ions can potentially be formed depending on which of the two hydrogen atoms is ejected during the fragmentation. The loss of a hydrogen atom on the aldehyde side is energetically much more favorable than the loss of $\mathrm{H}$ on the alkyne side, by $5.581 \mathrm{eV}$ according to our calculations (cf. Table 1). In this context, we present the optimized structures of the two considered $\mathrm{C}_{3} \mathrm{HO}^{+}$ions in Figure 5. We note that the experimental $\mathrm{AE}$ of the ion $m / z 53(11.26 \pm 0.03 \mathrm{eV})$ is in excellent agreement with the theoretically calculated energy of formation of the $\mathrm{HCCCO}^{+}\left(\mathrm{X}^{1} \Sigma^{+}\right)+\mathrm{H}$ fragments $(11.225 \mathrm{eV})$. The latter is only $5 \mathrm{meV}$ below the experimental finding. We can therefore unambiguously assign $m / z 53$ to the linear $\mathrm{HCCCO}^{+}+\mathrm{H}$ fragmentation channel (i.e. $\mathrm{H}$ abstraction on the aldehyde side). We note that the earlier electron impact ionization value from ref. ${ }^{14}(11.38 \pm 0.015 \mathrm{eV})$ is certainly too high, most probably because of the lower sensitivity of the used mass spectrometer which can be due to i) a lower 
number of ions formed in the source or ii) a poor transmission efficiency of the mass analyzer. Timmeticle Online of-flight mass analyzers, such as the one used in our work, are known for their excellent ion transmission efficiency close to $100 \%$ independent of mass. It is well known that lower ion detection sensitivity yields in general relatively higher AEs of cations formed by dissociative photoionization since reaction rates rise smoothly in the threshold region. It is furthermore well known that, in general, the determination of AEs by electron impact experiments is difficult. It is thus not surprising that the EI appearance energy of ref. ${ }^{14}$ is also less precise, by a factor of 5, compared to our measurements.

The experimental AE of $\mathrm{m} / \mathrm{z} 26(11.15 \pm 0.03 \mathrm{eV})$ is also in excellent agreement with the calculated $\mathrm{AE}$ value for the $\mathrm{HCCC}(\mathrm{O}) \mathrm{H} \rightarrow \mathrm{HCCH}^{+}+\mathrm{CO}$ fragmentation channel (cf. Fig. 5 for the optimized structure of $\mathrm{HCCH}^{+}$). The calculated $\mathrm{AE}$ falls inside the precision interval of the experimental AE determination for this cation. In the calculations, we also considered formation of a vinyl type $\mathrm{H}_{2} \mathrm{CC}^{+}$ cation potentially to be formed. This fragmentation channel is, however, off by $+1.785 \mathrm{eV}$ according to the calculations (cf. Table 1). We can therefore unambiguously assign $\mathrm{m} / z 26$ to an acetylene type cation. The EI ionization value from ref. ${ }^{14}(11.49 \pm 0.15 \mathrm{eV})$ can again be considered as too high, for the same reasons as outlined above.

The experimental AE of $m / z 29\left(\mathrm{HCO}^{+}\right)$is found at $13.4 \pm 0.3 \mathrm{eV}$. Considering the relatively large experimental error, this is in good agreement with the calculations which yield $\mathrm{AE}_{\text {calc }}=13.021 \mathrm{eV}$ considering the $\mathrm{HCCC}(\mathrm{O}) \mathrm{H} \rightarrow \mathrm{HCO}^{+}\left(\mathrm{X}^{1} \Sigma^{+}\right)+\mathrm{HC}_{2}\left(\mathrm{X}^{2} \Sigma^{+}\right)$channel. The AE of a $\mathrm{CO}^{+}$fragment $(\mathrm{m} / \mathrm{z}$ 28 ) is calculated to be $\mathrm{AE}_{\text {calc }}=13.80 \mathrm{eV}$. As noted above, this ion is however not observed in our measurements. The non-observation of a $\mathrm{HCCC}(\mathrm{O}) \mathrm{H} \rightarrow \mathrm{HCCH}+\mathrm{CO}^{+}$fragmentation channel is not surprising since it corresponds to the charge switch reaction of $\mathrm{HCCC}(\mathrm{O}) \mathrm{H} \rightarrow \mathrm{HCCH}^{+}+\mathrm{CO}$ yielding one of the two main fragments and being much lower in energy, by $2.68 \mathrm{eV}$, according to our calculations. The $\mathrm{HCCC}(\mathrm{O}) \mathrm{H} \rightarrow \mathrm{HCCH}+\mathrm{CO}^{+}$channel must therefore be of very low yield, if at all present. We finally note that this behavior is in full agreement with Stevenson's rule known from mass spectrometry textbooks. The AE from ref. ${ }^{14}(12.6 \pm 0.2 \mathrm{eV})$, determined with EI ionization mass spectrometry, must therefore be erroneous. Note that in the same paper the presented EI mass spectrum of propynal does not exhibit a $\mathrm{CO}^{+}$signal. The $\mathrm{CO}^{+}$ion is only observed with intense, pulsed $\mathrm{UV}$ laser radiation of $\mathrm{P}>5 \times 10^{7} \mathrm{~W} . \mathrm{cm}^{-2}$ as discussed above.

In the calculations we also considered the $\mathrm{HCCC}(\mathrm{O}) \mathrm{H} \rightarrow \mathrm{C}_{2}{ }^{+}\left({ }^{4} \Sigma_{\mathrm{g}}{ }^{+}\right)+\mathrm{H}_{2} \mathrm{CO}\left(\mathrm{X}^{1} \mathrm{~A}_{1}\right)$ and $\mathrm{HCCC}(\mathrm{O}) \mathrm{H} \rightarrow \mathrm{HC}_{2}{ }^{+}\left(\mathrm{X}^{1} \Sigma^{+}\right)$and $\mathrm{HCO}\left(\mathrm{X}^{2} \mathrm{~A}_{1}\right)$ fragmentation channels. Their AEs are calculated to be $\mathrm{AE}_{\text {calc }}=17.8667 \mathrm{eV}$ and $\mathrm{AE}_{\text {calc }}=19.104 \mathrm{eV}$, respectively. They both lie outside the energy range of our experiments and accordingly $\mathrm{C}_{2}{ }^{+}$and $\mathrm{HC}_{2}{ }^{+}$ions are not observed in the mass spectra we recorded.

\subsection{Excited states of the propynal cation}

In addition to the appearance energies of the parent and fragment cations that have been analysed above, the PEPICO spectra of $\mathrm{m} / \mathrm{z}, 54,53$ and 26 exhibit particular structures as can be seen in Figure 
6. First we note that the narrow lines, with decreasing intensity, that are seen in this spectrumbievardecle Online artefacts that shall not be considered in the discussion. The feature at $h v=11.834 \mathrm{eV}$ is an absorption line of Ar present in the gas filter of the beamline. It is used for energy calibration. The observed lines above $14 \mathrm{eV}$ correspond to positions of narrow autoionization features of molecular oxygen, present in the residual gas in the spectrometer chamber. They have high photoionization cross section and by consequence, the appearance of an intense $\mathrm{O}_{2}{ }^{+}$mass peak reduces the count rate of all other ions.

The PEPICO spectrum shown in Fig. 6 includes all hot electrons produced in the photoionization events since ion source extraction voltages have been chosen such that all electrons are detected by the position sensitive detector of DELICIOUS 3. The sum of the signals thus gives a total ion yield (TIY) spectrum that is proportional to the photoionization cross section of the molecule (not shown). The fractions of the $m / z 53,26$ signals represent the branching ratios of the respective fragmentation channels following the photoionization. Calibration of TIY spectra to absolute photoionization cross sections is important for astrophysical modeling applications. This will be done in future work using an appropriate calibration gas as has been achieved earlier by some of us (see for example. ref. ${ }^{46}$ ). We note that mixing the sample with a calibration gas, at a well-known partial pressure, is difficult with fragile molecules such as propynal.

The observed broad bands in the PEPICO spectrum will be analyzed in the following. In this context we also present the TPEPICO spectra of the three major ions in Figure 7. This experimental spectrum consists of two parts recorded separately (AIE up to $13 \mathrm{eV}$ and $13 \mathrm{eV}$ to $15.75 \mathrm{eV}$ ). The intensity of the higher energy part has been multiplied with a factor of 5 in order to better visualize the observed structure. In total, four large bands are observed in Fig. 7, in accordance with the earlier photoelectron spectra. ${ }^{10,40}$ In order to understand their origin we calculated the energy levels of excited states of the propynal cation in the energy range of the experiments. In a first step, an orbital analysis was carried out to identify the outermost molecular orbitals of neutral propynal (cf. Table 2). The highest occupied molecular orbital, HOMO, (12 $\left.\mathrm{a}^{\prime}\right)$ corresponds to the lone pair of the $\mathrm{O}$ atom. The lowest unoccupied molecular MO, LUMO, (3a") is a $\pi$-type anti bounding orbital spreading over the whole molecule. The HOMO-1 (2a"), HOMO-2 (11a') and HOMO-3 (1a") MOs correspond to $\pi$-type orbital localised on the lone pair of $\mathrm{O}$ and of $\mathrm{C} \equiv \mathrm{C}, \sigma$-type $\mathrm{HCO}$ orbital and $\pi$-bonding orbital respectively.

The excited electronic states of the propynal cation were computed using the MRCI method on top of state-averaged multiconfigurational CASSCF computations. They are computed at the equilibrium geometry of the propynal ${ }^{+}$ion $\left(\widetilde{\mathrm{X}}^{2} \mathrm{~A}^{\prime}\right)$. The results from the MRCI calculations are summarized in Table 3, together with experimentally observed vertical energies from the TPEPICO spectrum. Table 3 also lists their dominant electron configurations as quoted at the propynal ${ }^{+}$ion $\left(\widetilde{\mathrm{X}}^{2} \mathrm{~A}^{\prime}\right)$ equilibrium. The MRCI results are also shown in Fig. 7 as bars and vertical dotted lines are inserted to guide the eye. 


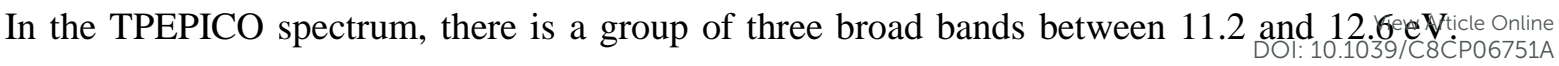
They are only seen in the $\mathrm{m} / z 53$ and $\mathrm{m} / z 26$ spectra. We can thus deduce that even at these energies the parent ion is formed preferentially in the ground state. The $m / z 26$ spectrum shows a first maximum at $11.41 \pm 0.02 \mathrm{eV}$, thus $0.69 \pm 0.02 \mathrm{eV}$ above the ion ground state. This experimental value compares favorably with the energy of the $1^{2} \mathrm{~A}$ " state found in the calculations at $0.65 \mathrm{eV}$ (cf. Table 3 and Fig. 7). It is obtained after removal of one electron from the ( $\left.2 \mathrm{a}^{\prime \prime}\right) \mathrm{MO}$ of neutral propynal. We therefore assign the TPEPICO peak to the vertical transition to the $1^{2} \mathrm{~A}$ " state. The maximum of the second peak in this part of the spectrum is located at $11.53 \pm 0.03 \mathrm{eV}$, thus $0.81 \pm 0.03 \mathrm{eV}$ above the ground state. It is found in both main fragmentation channels. We assign this peak to the vertical transition to the $2{ }^{2} \mathrm{~A}$ ' state of the cation found in the calculations (cf. Table 3 and Fig. 7). It is due to the removal of one electron from the $11 \mathrm{a}^{\prime}$ MO. It is remarkable that these two close-lying electronic states obtained with the MRCI calculations coincide very well with the measurement. They may well interact by vibronic coupling and hence the mixing of their respective electronic wave functions. Therefore, we expect a prominent contribution of predissociation processes and vibronic interaction. Predissociation seems indeed to predominate since the population of the two states is only seen in the fragmentation channels (cf. Fig. 7). Parent ion formation is not observed from the $1^{2} \mathrm{~A}^{\prime \prime}$ and $2{ }^{2} \mathrm{~A}^{\prime}$ states. The vibronic interactions between the two states probably participate to the congestion of the bands. The experimentally observed structure between 11.7 and $12.0 \mathrm{eV}$, on the descending flank of the TPEPICO band at $11.53 \mathrm{eV}$, is therefore difficult to assign to a distinct vibronic structure and by consequence we do not attempt this.

Between 13.8 and $15.75 \mathrm{eV}$ additional large bands are observed in the TPEPICO spectrum of $\mathrm{m} / \mathrm{z} 53$ and 26. Also in this region, the parent ion $\mathrm{m} / \mathrm{z} 54$ is only formed in its ground state since its TPEPICO signal is zero. The $\mathrm{m} / \mathrm{z} 53,26$ bands are much weaker in intensity than the bands corresponding to the ground and the first two excited states. We can distinguish two bands at most, the first one peaking at about $14.45 \pm 0.1 \mathrm{eV}$ and the second at about $14.8 \pm 0.1 \mathrm{eV}$, respectively $3.74 \pm 0.1 \mathrm{eV}$ and $4.1 \pm 0.1 \mathrm{eV}$ above the ground state. In agreement with our measurements, a high density of electronic states are found in the calculations since four excited states are computed between 14.3 and $15.0 \mathrm{eV}\left(2^{2} \mathrm{~A}^{\prime \prime} ; 3{ }^{2} \mathrm{~A}^{\prime \prime} ; 3{ }^{2} \mathrm{~A}^{\prime} ; 4{ }^{2} \mathrm{~A}^{\prime \prime}\right)$ i.e. at $3.65,4.06,4.13$ and 4.30 above $\mathrm{C}_{3} \mathrm{H}_{2} \mathrm{O}^{+}\left(\mathrm{X}^{2} \mathrm{~A}^{\prime}\right)$. Thus, they are probably strongly coupled, leading again to congestion and predissociation, in agreement with what is observed experimentally.

Finally, we observe a weak large band for $m / z 53$ (but not in $m / z$ 26) spectrum expanding from 13 to $13.8 \mathrm{eV}$ range and peaking at about $13.4 \pm 0.1 \mathrm{eV}$ (c. Fig. 7). No electronic states of popynal ${ }^{+}$are computed in this energy range. From the analysis of the 3-dimensional photoelectron intensity matrix (PEPICO counts as a function of photon and electron kinetic energy) it can also be concluded that autoionization of propynal plays no role in the whole energy range of our study. We thus performed MRCI computations of linear $\mathrm{HCCCO}^{+}$(not shown here) since this band might arise from the population of excited states of $\mathrm{HCCCO}^{+}$. According to these calculations they are, however, located at $>4 \mathrm{eV}$ above 
the corresponding ground state. Instead, we suggest strong rearrangements of the preliminary formed ${ }_{D O 1}$ : $10.1039 / C 8 C P 06751 \mathrm{~A}$ linear $\mathrm{HCCCO}^{+}$possibly leading to other isomers of this fragment cation. This remains to be clarified in the future.

\subsection{Tentative assignment of the vibrational structure of the ground state TPEPICO spectrum}

The PBE0 anharmonic frequencies of the 12 vibrational modes of the propynal ${ }^{+}$ion in its electronic ground state are listed in Table 4. These frequencies are arranged in a descending order under $\mathrm{a}^{\prime}$ symmetry (for the 9 in-plane vibrations) and a" symmetry (for the 3 out-of-plane vibrations). The molecular structures of the neutral propynal and its cation, both shown in Figure 8, are very close. Only small changes can be noticed leading to short vibrational progressions for the propynal ${ }^{+}\left(\mathrm{X}^{2} \mathrm{~A}^{\prime}\right)+\mathrm{e}^{-} \leftarrow$ propynal $\left(\mathrm{X}^{1} \mathrm{~A}^{\prime}\right)+\mathrm{h} v$ transition. This is in line to the removal of one electron form the unbounding $12 \mathrm{a}^{\prime \prime}$ MO (Table 2) upon ionization of neutral propynal.

Notably, the $\mathrm{HCO}$ bond angle of the aldehyde group changes from $121.6^{\circ}$ to $113.8^{\circ}$ and the bond length of the C-C single bond changes from $1.454 \AA$ to $1.391 \AA$. Other geometrical parameters stay almost constant. Based on these changes we can tentatively assign the vibrational structure observed in the ground state TPEPICO spectrum (cf. Fig. 2). Correspondingly, we propose excitation of the C-H rocking mode vibration $v_{5}$ (up to 3 quanta). Its anharmonic frequency $E_{a h}$ is calculated to be $E_{a h}=$ $1129 \mathrm{~cm}^{-1}$. In parallel, we propose that excitation of the $\mathrm{C}-\mathrm{H}$ wagging mode vibration $v_{6}\left(\mathrm{E}_{\mathrm{ah}}=996 \mathrm{~cm}^{-}\right.$ ${ }^{1}$; up to 3 quanta) takes place as well. The change in the HCO bond angle leads very likely to the excitation of the $\mathrm{C}=\mathrm{O}$ stretching mode $v_{4}\left(\mathrm{E}_{\mathrm{ah}}=1527 \mathrm{~cm}^{-1}\right.$; up to 2 quanta $)$ and a $\mathrm{CCO}$ in-plane bending mode $v_{8}$ that is potentially excited with up to 8 quanta $\left(E_{a h}=417 \mathrm{~cm}^{-1}\right)$. In Fig. 2 one can also recognize that the $0_{0}{ }^{0}$ band has a slight shoulder on the higher energy side. This could be due to the excitation of the low frequency mode $v_{9}\left(E_{\text {ah }}=144 \mathrm{~cm}^{-1}\right)$ corresponding to a $\mathrm{C}-\mathrm{C} \equiv \mathrm{C}$ in-plane bending vibration. All these modes are of $\mathrm{a}^{\prime}$ symmetry and thus the population of these bands are allowed by single photoionization rules. Therefore, the TPEPICO spectrum of Figure 2 is fully assigned by the consideration of these four vibrational progressions.

\section{Conclusion}

We studied the single photon ionization and dissociative photoionization of propynal from its AIE up to $15.75 \mathrm{eV}$ both experimentally and theoretically. Experiments have been carried out using the DELICIOUS3 spectrometer in connection with the DESIRS beamline at SOLEIL. Mass spectra, PEPICO and TPEPICO spectra have been interpreted using a combination of ab-initio and DFT calculations, with a calibrated methodological approach. This methodological approach relies on powerful computations performed on the ground states of propynal and propynal ${ }^{+}$and on the excited states of the cationic molecule. Herein, geometry optimizations were carried out at the (R)CCSD(T)F12/aVTZ level of theory. Using such a theoretical approach the calculation of the $\mathrm{E}_{\mathrm{corr}}$ as the sum of 
$\mathrm{E}_{(\mathrm{R}) \mathrm{CCSD}(\mathrm{T})-\mathrm{F} 12}$ and the $\mathrm{CV}, \mathrm{SR}$ and ZPE corrections gives AIE of $10.717 \mathrm{eV}$ in excellent agreementiwithicle Online the experimental value of $10.715 \pm 0.005 \mathrm{eV}$. The latter value has been obtained from the $0_{0}^{0}$ transition of the TPEPICO spectrum of the parent ion ground state. It is more precise, by a factor of 30 , than a previously available electron impact ionization measurement. Fragmentation thresholds have been theoretically calculated. This allowed the assignment of experimentally observed masses to the formation of $\mathrm{HC}_{3} \mathrm{O}^{+}\left(\mathrm{X}^{1}{ }^{1 \Sigma}\right), \mathrm{C}_{2} \mathrm{H}_{2}{ }^{+}\left(\mathrm{X}^{2} \Pi\right)$ and $\mathrm{HCO}^{+}\left(\mathrm{X}^{1} \Sigma^{+}\right)$formed by dissociative photoionization reactions. The calculated appearance energies are in excellent agreement with the experimentally determined formation thresholds of ions at $\mathrm{m} / \mathrm{z} 53,26$ and 29, respectively. The appearance energies have been measured for the first time using photoionization. They are more accurate and precise than previously available values from electron impact ionization. MRCI calculations suggest the existence of seven ionic excited states in the energy range of the measurements. This may lead to spectral congestion and explains the absence of vibronic structure of the excited state bands in the observed spectra. The ground state spectrum of the ion, however, exhibits a well resolved vibrational structure that has been satisfactorily analyzed taking advantage of DFT/PBE0 calculations of the anharmonic frequencies of the 12 vibrational modes of the propynal cation.

Overall, our ab-initio calculations are fully consistent with the present set of experimental data. From a methodological point view, our work confirms the good performance of the (R)CCSD(T)-F12 $+\Delta \mathrm{CV}+\Delta \mathrm{SR}+\Delta \mathrm{ZPE}$ composite scheme for the accurate derivation of the energetics of medium-sized molecules of interest in astrophysics. ${ }^{47}$ The validation consists of a direct comparison between the values of experimental ionization energies and fragmentation thresholds, and energies calculated theoretically. Moreover, our calculations allowed for an in depth interpretation of the experiments. The present results will be useful to interpret astrophysical observations of the propynal cation in various objects of the interstellar medium. Photoionization cross section data (after proper calibration of the total ion yield spectra measurement in our work) as well as branching ratios of dissociative photoionization reactions, all as function of the photon energy, should be included into astrochemical databases in the future.

\section{Acknowledgement}

This work was supported by the Programme National "Physique et Chimie du Milieu Interstellaire" (PCMI) of CNRS/INSU with INC/INP co-funded by CEA and CNES. We thank the COST ACTION CM 1401 "Our Astro-chemical history" and COST ACTION CM 1405 "Molecules in Motions" for support. We are indebted to the general technical staff of Synchrotron SOLEIL for running the facility under proposals 20141309 and 20170203. J.-C. G. thanks the Centre National d'Etudes Spatiales for a financial support. We would like also to thank Jean-François Gil for his technical help on the SAPHIRS molecular beam chamber. We thank Ayad Bellili, Lionel Poisson, Nicolas Fray, Yves Bénilan, Dušan Božanić and Gustavo Garcia for precious help during the measurement campaigns at SOLEIL. 
${ }^{1}$ V. Wakelam, E. Herbst, J. C. Loison, I. W. M. Smith, V. Chandrasekaran, B. Pavone,... \& V. M. Bierbaum,. A kinetic database for astrochemistry (KIDA), Astrophys. J., Suppl. Ser., 2012, 199, 21.

${ }^{2}$ W. M. Irvine, R. D. Brown, D. M. Cragg, P. Friberg, P. D. Godfrey, N. Kaifu, H. E. Matthews, M. Ohishi, H. Suzuki, H. Takeo, A new interstellar polyatomic molecule: detection of propynal in the cold cloud TMC-1, Astrophys. J., 1988, 335, L89L93.

${ }^{3}$ N. G. Adams, D. Smith, K. Giles, E. Herbst, E., The production of C (n) O, HC (n) O, and H2C (n) O molecules in dense interstellar clouds, Astron. Astrophys., 1989, 220, 269-271.

${ }^{4}$ J. M. Hollis, P. R. Jewell, F. J. Lovas, A. Remijan, H. Møllendal, Green Bank Telescope Detection of new interstellar aldehydes: propenal and propanal, Astrophys. J., 2004, 610, L21-L24.

${ }^{5}$ R. A. Loomis, B. A. McGuire, C. Shingledeckker, C. H. Johanson, S. Blair, A. Robertosn, A. J. Remijan, Investigating the minimum energy principle in searches for new molecular species - the case of the $\mathrm{H}_{2} \mathrm{C}_{3} \mathrm{O}$ isomers. Astrophys. J., 2015, 799, 34.

${ }^{6}$ J. C. Loison, M. Agúndez, N. Marcelino, V. Wakelam, K. M. Hickson, J. Cernicharo,... \& M. Guélin, The interstellar chemistry of $\mathrm{H}_{2} \mathrm{C}_{3} \mathrm{O}$ isomers, Mon. Not. R. Astron. Soc., 2016, 456, 4101-4110.

${ }^{7}$ L. Zhou, R. I. Kaiser, L. Gyun Gao, A. H. H. Chang, M.-C. Liang, Y. L. Yung, Pathways to oxygen-bearing molecules in the interstellar medium and in planetary atmospheres: cyclopropenone $\left(\mathrm{c}-\mathrm{C}_{3} \mathrm{H}_{2} \mathrm{O}\right)$ and propynal (HCCCHO), Astrophys. J. 2008, 686, 1493-1502.

${ }^{8}$ D. Semenov, F. Hersant, V. Wakelam, A. Dutrey, E. Chapillon, St. Guilloteau, Th. Henning, R. Launhardt, V. Piétu and K. Schreyer, Chemistry in disks - IV. Benchmarking gas-grain chemical models with surface reactions, Astron. Astrophys. 2010, 522, A42.

${ }^{9}$ V. A. Krasnopol'Skii, C3 and CN parents in Comet P/Halley, Astron. Astrophys., 1991, 245, 310-315.

${ }^{10}$ P. Carlier, G. Mouvier, D. Mesnard, L. Miginiac, Etude par spectrométrie de photoélectrons de la structure électronique des enynes conjugués, J. Electron Spectrosc. Relat. Phenom., 1979, 16, 147-167.

${ }^{11}$ A. Komornicki, C. E. Dykstra, M. A. Vincent, L. Radom, L., A theoretical study of propadienone and its isomers propynal and cyclopropenone. J. Am. Chem. Soc. 1981, 103, 1652-1656.

${ }^{12}$ G. Bouchoux, Y. Hoppilliard, J. P. Flament, Structures and stabilities of [C3H3O]+ ions in the gas phase: A molecular orbital study. Org. Mass Spectrom., 1985, 20, 560-564.

${ }^{13}$ P. Russegger, J. R. Huber, Photodissociation dynamics of propynal (HCCCHO) and butynal (H3C-CCCHO). Ab initio and RRKM calculations. Chem. Phys., 1984, 89, 33-42.

${ }^{14}$ J. Opitz, Photoionization of propynal in the gas phase, Int. J. Mass Spectrom. Ion Processes, 1991, 107, 503-513.

${ }^{15}$ J. L. Holmes, K. J. Jobst, J. K. Terlouw, Small (poly) unsaturated oxygen containing ions and molecules: a brief assessment of their thermochemistry based on computational chemistry, Eur. J. Mass Spectrom., 2009, 15, 261-273.

${ }^{16}$ K. I. Öberg, Photochemistry and astrochemistry: Photochemical pathways to interstellar complex organic molecules, Chem. Rev., 2016, 116, 9631-9663.

${ }^{17}$ L. Nahon, N. de Oliveira, G.A. Garcia, J.-F. Gil, N. Pilette, O. Marcouillé, B. Largarde, F. Polack, DESIRS : a state-of-theart VUV beamline featuring high resolution and variable polarization for spectroscopy and dichroism at SOLEIL, $J$. Synchrotron Rad., 2012, 19, 508-520.

${ }^{18}$ G. A. Garcia, B. K. Cunha de Miranda, M. Tia, S. Daly, L. Nahon, DELICIOUS III: a multipurpose double imaging particle coincidence spectrometer for gas phase vacuum ultraviolet photodynamics studies, Rev. Sci. Instr., 2013, 84, 053112.

${ }^{19}$ J. C. Sauer, Propiolaldehyde, Org. Synth., 1956, 4, 813 and 1963, 36, 66.

${ }^{20}$ M.J. Frisch, G. W. Trucks, H. B. Schlegel, G. E. Scuseria, M. A. Robb, J. R. Cheeseman,... \& H. Nakatsuji, Gaussian 09, Revision E. 01, 2009, Gaussian. Inc., Wallingford CT.

${ }^{21}$ H.-J. Werner, P. J. Knowles, G. Knizia, F. R. Manby, M. Schütz, P. Celani, T. Korona, R. Lindh, A. Mitrushenkov and G. Rauhut, et al., MOLPRO, ab initio programs package, 2015, http://www.molpro.net.

${ }^{22}$ M. Hochlaf, Y. Pan, K. C. Lau, Y. Majdi, L. Poisson, G. A Garcia, L. Nahon, M. Mogren Al Mogren, M. Schwell, Vibrationally resolved photoelectron spectroscopy of electronic excited states of DNA bases: Application to the $\tilde{A}$ state of thymine cation, J. Phys. Chem. A, 2015, 119, 1146-1153. 
${ }^{23}$ Y. Majdi, M. Hochlaf, Y. Pan, K.C. Lau, L. Poisson, G. A. Garcia, L. Nahon, M. Mogren Al Mogren, M. Schwell, Theoretical and experimental photoelectron spectroscopy characterization of the ground state of thymine cation, J. Phys. Chem. A, 2015, 119, 5951-5958.

${ }^{24}$ T. B. Adler, G. Knizia and H.-J. Werner, A simple and efficient CCSD(T)-F12 approximation, J. Chem. Phys., 2007, 127, 221106

${ }^{25}$ G. Knizia, T. B. Adler and H.-J. Werner, Simplified CCSD(T) - F12 methods: theory and benchmarks, J. Chem. Phys., 2009, 130, 054104.

${ }^{26}$ T. B. Adler, H.-J. Werner, F. R. Manby, Local explicitly correlated second-order perturbation theory for the accurate treatment of large molecules, J. Chem. Phys., 2009, 130, 054106.

${ }^{27}$ T. B. Adler and H.-J. Werner, Local explicitly correlated coupled-cluster methods: Efficient removal of the basis set incompleteness and domain errors, J. Chem. Phys., 2009, 130, 241101.

${ }^{28}$ R. A. Kendall, T. H. Dunning Jr, R. J. Harrison, Electron affinities of the first- row atoms revisited. Systematic basis sets and wave functions, J. Chem. Phys., 1992, 96, 6796-6806.

${ }^{29}$ J.D. Watts, J. Gauss, R. J. Bartlett, Coupled- cluster methods with noniterative triple excitations for restricted open- shell Hartree-Fock and other general single determinant reference functions. Energies and analytical gradients, J. Chem. Phys., 1993, 98, 8718-8733.

${ }^{30}$ T. H. Dunning, Gaussian Basis Sets for Use in Correlated Molecular Calculations. I. The Atoms Boron Through Neon and Hydrogen, J. Chem. Phys., 1989, 90, 1007-1023.

${ }^{31}$ M. Douglas, N. M. Kroll, Quantum electrodynamical corrections to the fine structure of helium, Ann. Phys., 1974, 82, 89155 .

${ }^{32}$ G. Jansen, B. A. Heß, Revision of the Douglas-Kroll transformation, Phys. Rev. A, 1989, 39, 6016.

${ }^{33}$ W. A. De Jong, R. J. Harrison, D. A. Dixon, Parallel Douglas-Kroll energy and gradients in NWChem: estimating scalar relativistic effects using Douglas-Kroll contracted basis sets. J. Chem. Phys., 2001, 114, 48-53.

${ }^{34}$ C. Adamo and V. Barone, Toward Reliable Density Functional Methods Without Adjustable Parameters: The PBE0 Model, J. Chem. Phys., 1999, 110, 6158-6170.

${ }^{35}$ P. J. Knowles, H. J. Werner, An efficient second-order MC SCF method for long configuration expansions, Chem. Phys. Lett., 1985, 115, 259-267.

${ }^{36}$ H. J. Werner, P. J. Knowles, A second order multiconfiguration SCF procedure with optimum convergence, J. Chem. Phys., 1985, 82, 5053-5063.

${ }^{37}$ H. J. Werner, P. J. Knowles, An efficient internally contracted multiconfiguration-reference configuration interaction method, J. Chem. Phys., 1988, 89, 5803-5814.

${ }^{38}$ P. J. Knowles and H.-J. Werner, An efficient method for the evaluation of coupling coefficients in configuration interaction calculations, Chem. Phys. Lett., 1988, 145, 514-522.

${ }^{39}$ K. R. Shamasundar, G. Knizia and H.-J. Werner, A new internally contracted multi-reference configuration interaction method, J. Chem. Phys., 2011, 135, 054101.

${ }^{40}$ W. Von Niessen, G. Bieri, L. Asbrink, 30.4-nm He(II) photoelectron spectra of organic molecules. II Oxo-compounds (C,H,O), J. Electron. Spectrosc. Rel. Phenom. 1980, 21, 175-191.

${ }^{41}$ S. Rayne, K. Forest, Thermochemistry of mono-and disubstituted acetylenes and polyynes at the Gaussian-4 level of theory, Comput. Theor. Chem., 2011, 970, 15-22.

42 Y. Pan, K.-C. Lau, L. Poisson, G. A. Garcia, L. Nahon and M. Hochlaf. Slow Photoelectron Spectroscopy of 3Hydroxyisoquinoline. J. Phys. Chem. A, 2013, 117, 8095.

${ }^{43}$ A. Bellili, M. Schwell, Y. Bénilan, N. Fray, M.-C. Gazeau, M. Mogren Al-Mogren, J.-C. Guillemin, L. Poisson and M. Hochlaf, VUV photoionization and dissociative photoionization of the prebiotic molecule acetyl cyanide: Theory and experiment, J. Chem. Phys., 2014, 141, 134311.

${ }^{44}$ A. Bellili, M. Schwell, Y. Bénilan, N. Fray, M.-C. Gazeau, M. Mogren Al-Mogren, J.-C. Guillemin, L. Poisson and M. Hochlaf, VUV photoionization and dissociative photoionization spectroscopy of the interstellar molecule aminoacetonitrile: Theory and experiment, J. Mol. Spectrosc., 2015, 315, 196.

${ }^{45}$ Z. Chen, K.-C. Lau, G. A. Garcia, L. Nahon, D. K. Božanić, L. Poisson, M. Mogren Al-Mogren, M. Schwell, J. S. Francisco, A. Bellili and M. Hochlaf, Identifying cytosine-specific isomers via high-accuracy single photon ionization, J. Am. Chem. Soc., 2016, 138, 16596.

${ }^{46}$ S. Leach, G. A. Garcia, Y. Bénilan, N. Fray, M. C. GłAeau, F. Gaie-Levrel, N. Champion, M. Schwell, Ionization photophysics and spectroscopy of cyanoacetylene, J. Chem. Phys., 2014, 140,184304. 
${ }^{47}$ M. Hochlaf, Advances in spectroscopy and dynamics of small and medium sized molecules and clusters, Phys. Chem. Chem. Phys., 2017, 19, 21236-21261. 


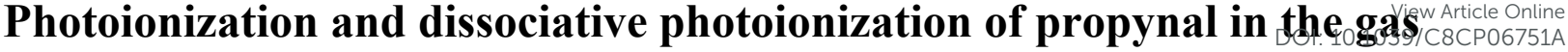 phase: Theory and experiment}

Imene Derbali, ${ }^{1}$ Helgi Rafn Hrodmarsson, ${ }^{2}$ Zied Gouid, ${ }^{3}$ Martin Schwell,,${ }^{4,}$ Marie-Claire Gazeau, ${ }^{4}$ Jean-Claude Guillemin, ${ }^{5}$ Majdi Hochlaf, ${ }^{3 *}$ Mohammad Esmaïl Alikhani, ${ }^{1}$ Emilie-Laure Zins ${ }^{1 *}$

Figures and Tables 


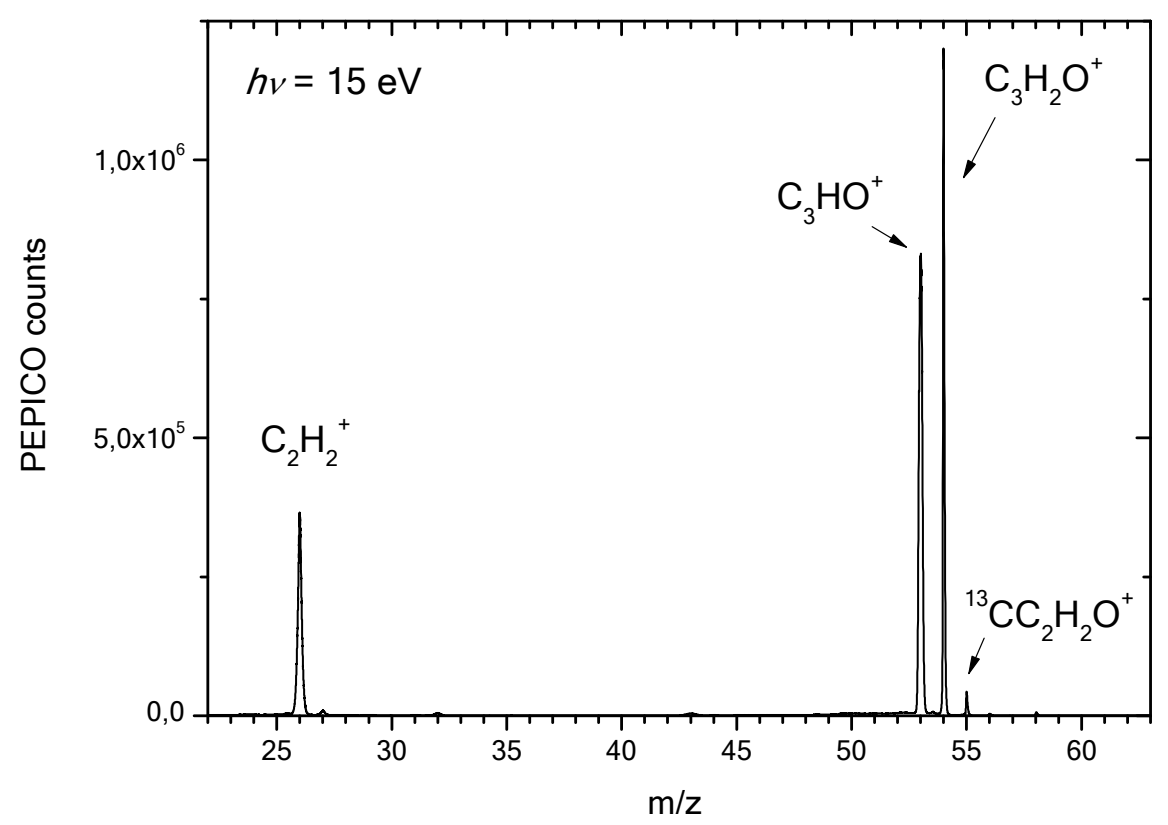

Figure 1: Time-of-flight mass spectrum of propynal photoionized at $h v=15 \mathrm{eV}$. 


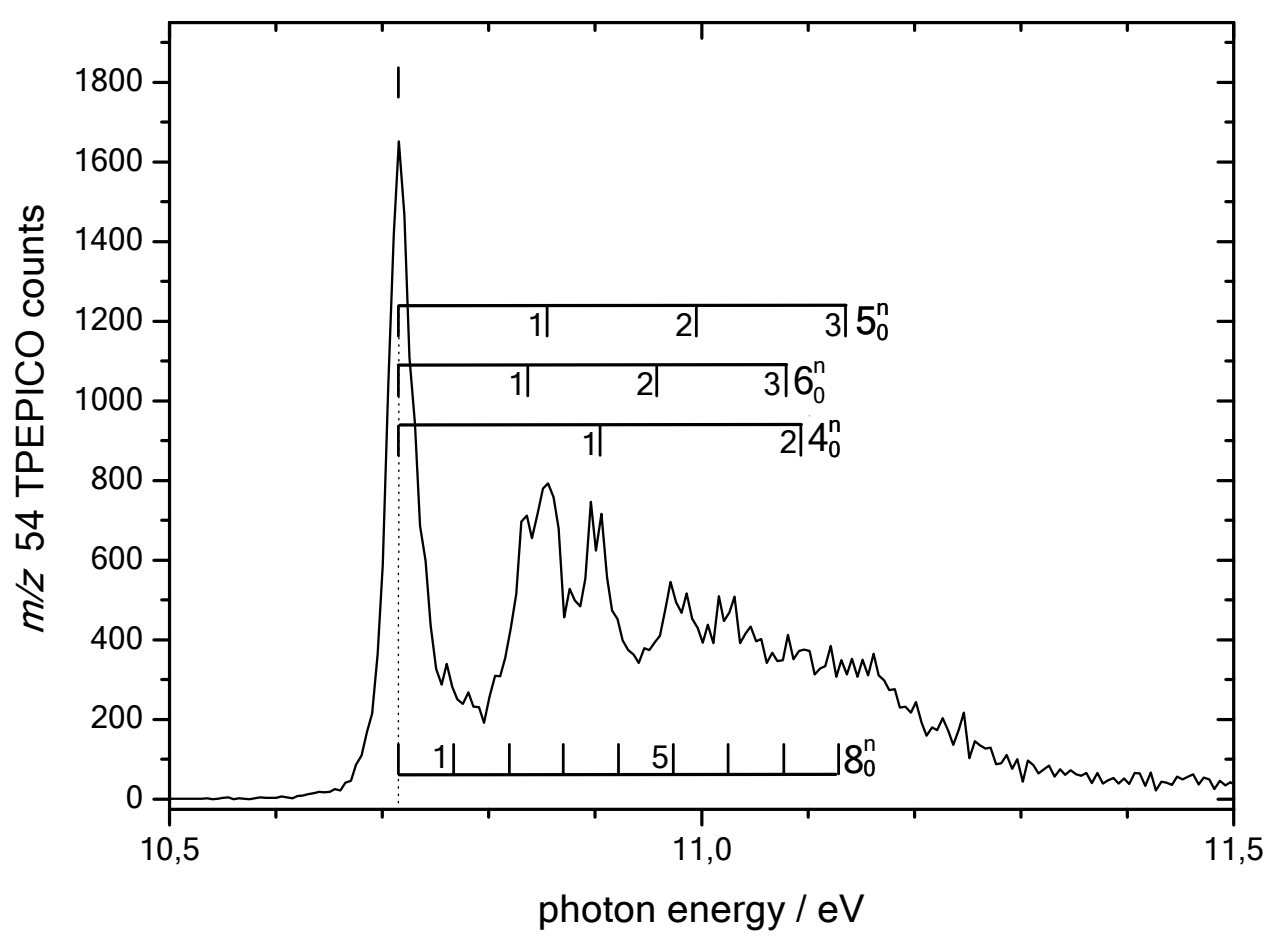

Figure 2: TPEPICO spectra of the propynal parent ion (m/z 54). The experimental adiabatic ionization energy (AIE) is taken as the median of the $\mathbf{0}_{\mathbf{0}}^{\mathbf{0}}$ band (indicated by a bar and a vertical dotted line). The observed vibrational structure is discussed in paragraph 3.4. 

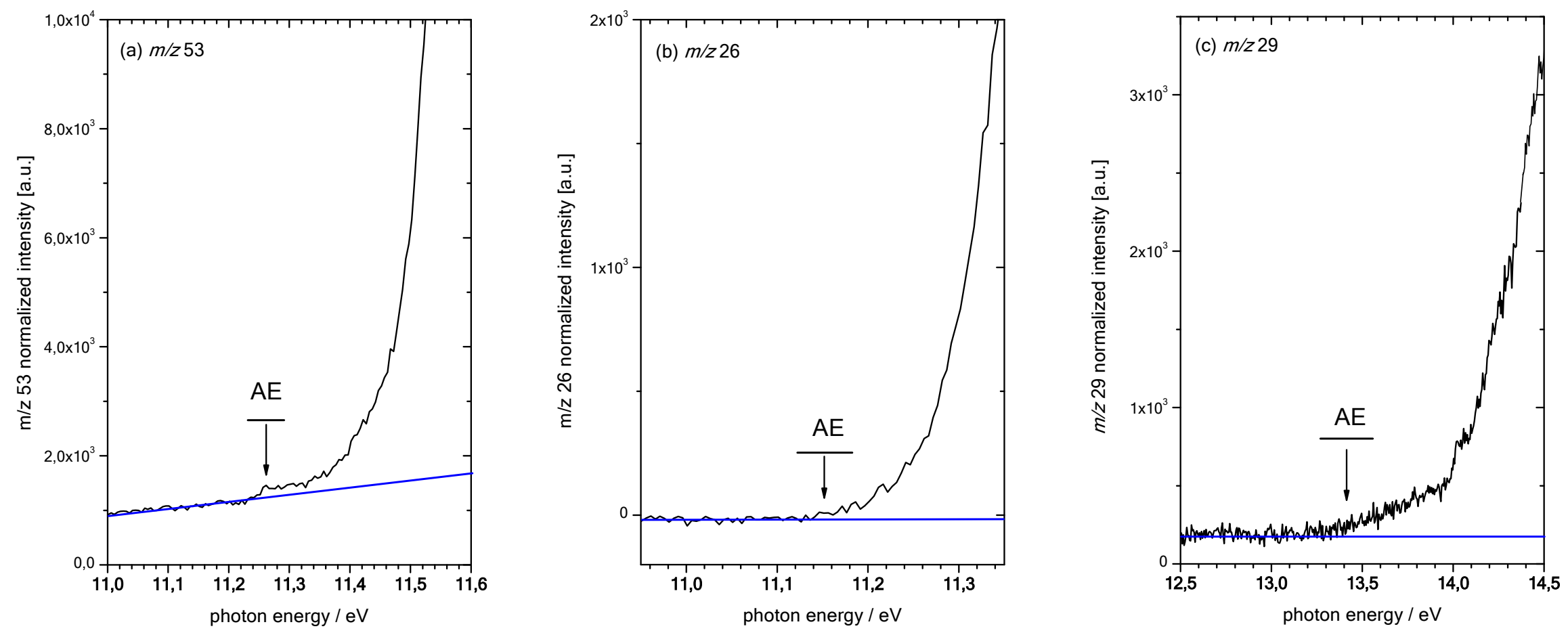

Figure 3: PEPICO spectra of the three observed propynal fragments formed by dissociative photoionization. (a) $m / z 53$ (b) $m / z 26$, (c) $m / z 29$. Solid blue lines are inserted to indicate the base line level. Appearance energies were set to the energy where the mass signal exceeds the noise level of the baseline. Horizontal bars are inserted to indicate the estimated error of the deduced AE. 


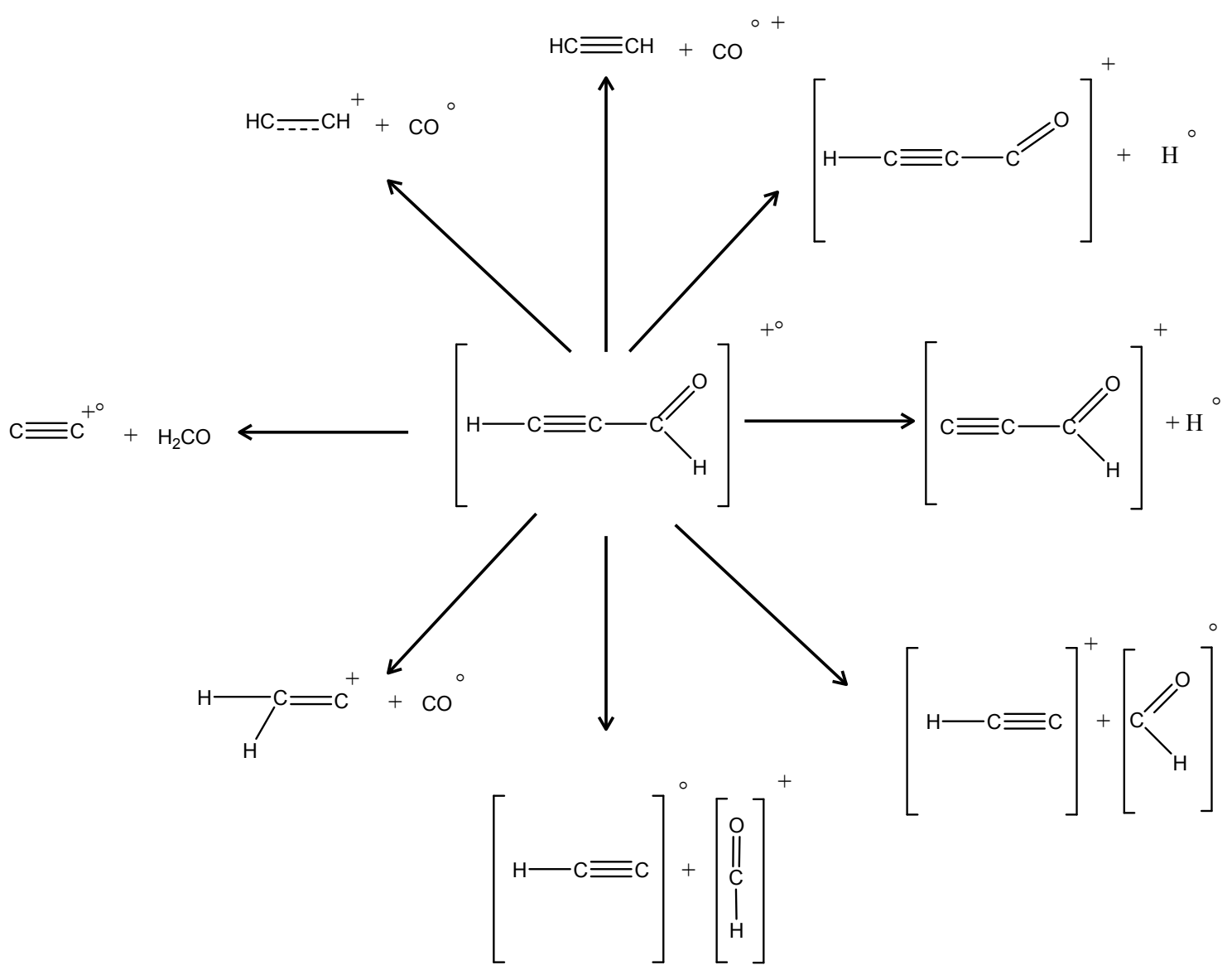

Figure 4: Fragmentation pathways considered for the quantum-chemical calculations. 


$$
\mathrm{C}_{3} \mathrm{HO}^{+}\left(\mathrm{X}^{1} \Sigma^{+}\right)
$$

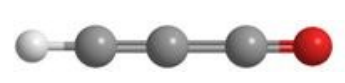

$\mathrm{C}_{3} \mathrm{HO}^{+}\left(\mathrm{X}^{1} \mathrm{~A}^{\prime}\right)$

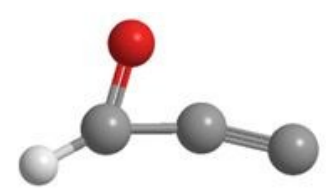

$\mathrm{C}_{2} \mathrm{H}_{2}{ }^{+}\left(\mathrm{X}^{2} \mathrm{~A}_{1}\right)$

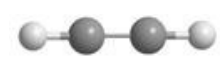

Figure 5: Optimized structures of two isomers of $\mathrm{C}_{3} \mathrm{HO}^{+}$and of $\mathrm{C}_{2} \mathrm{H}_{2}{ }^{+}$ions, at the (R)CCSD(T)-F12 level using the using the aug-cc-pVTZ basis set. 


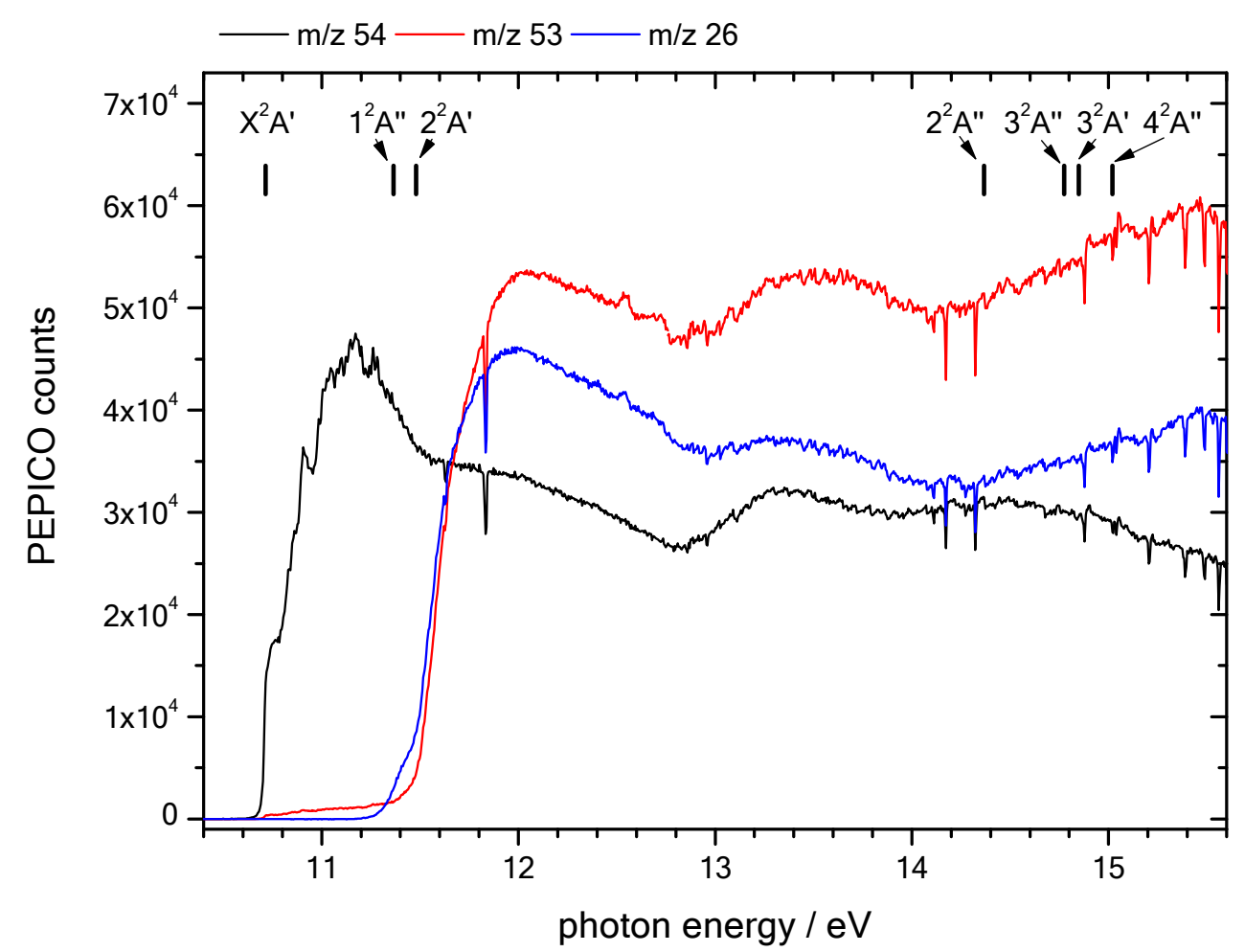

Figure 6: PEPICO spectra of the propynal parent ion $\mathrm{m} / \mathrm{z} 54$ (black line) and the two observed main fragments: $m / z 53$ (red line) and $m / z 26$ (blue line) formed by dissociative ionization energy in the 10.4-15.75 eV energy range. Narrow features in the spectra are artefacts (see text for details). Bars indicate the results from MRCI calculations of the excited states of propynal (cf. Table 3). 


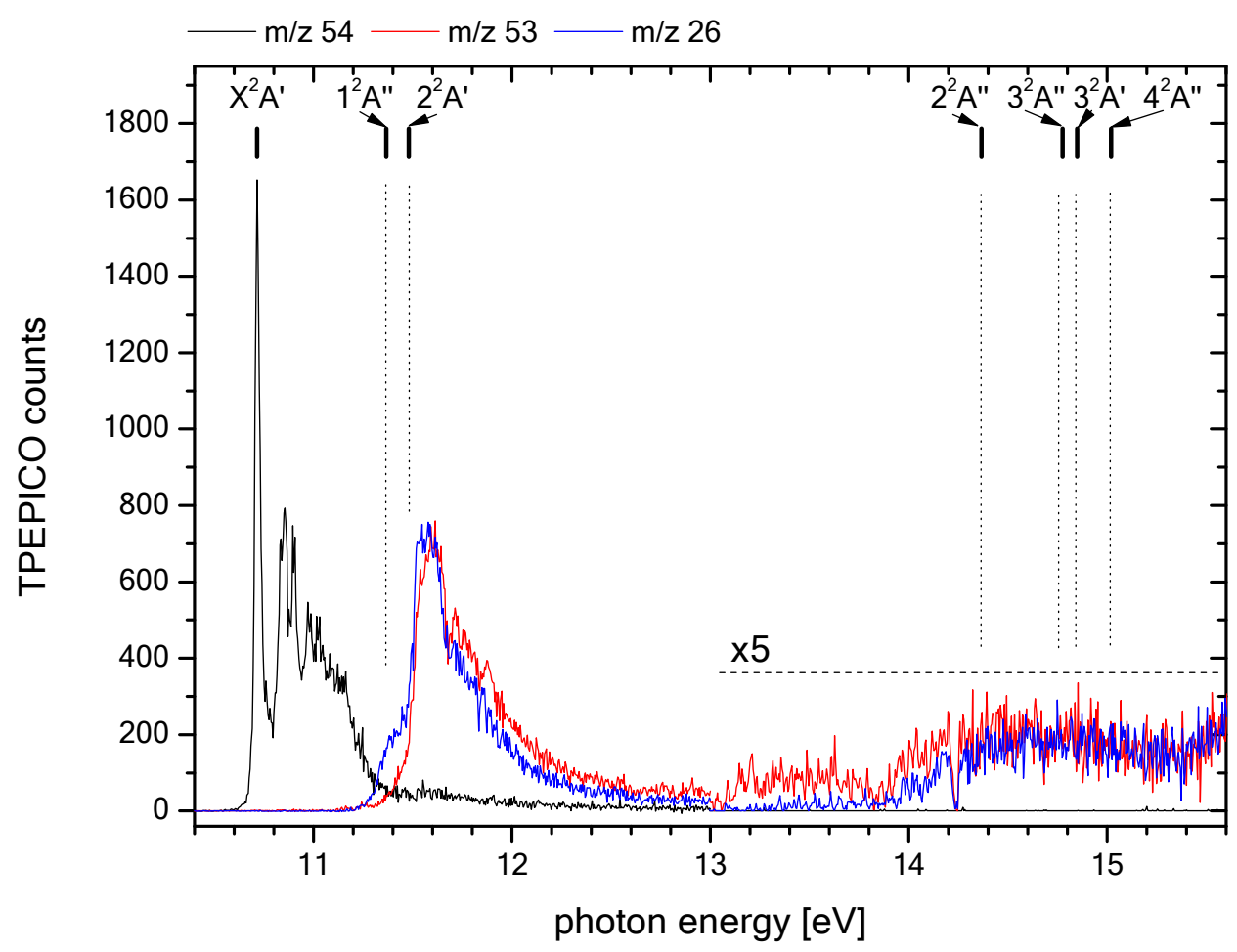

Figure 7: TPEPICO spectra of the propynal parent ion $\mathrm{m} / z 54$ (black line) and the two observed main fragments: $m / z 53$ (red line) and $m / z 26$ (blue line) formed by dissociative ionization energy in the 10.4-15.75 eV energy range. In the energy range $\mathrm{E}>13 \mathrm{eV}$, the original intensity has been multiplied by a factor of 5 . Solid bars indicate the results from MRCI calculations of the excited states of propynal (same as Fig. 6). Dotted lined are inserted to guide the eye. 

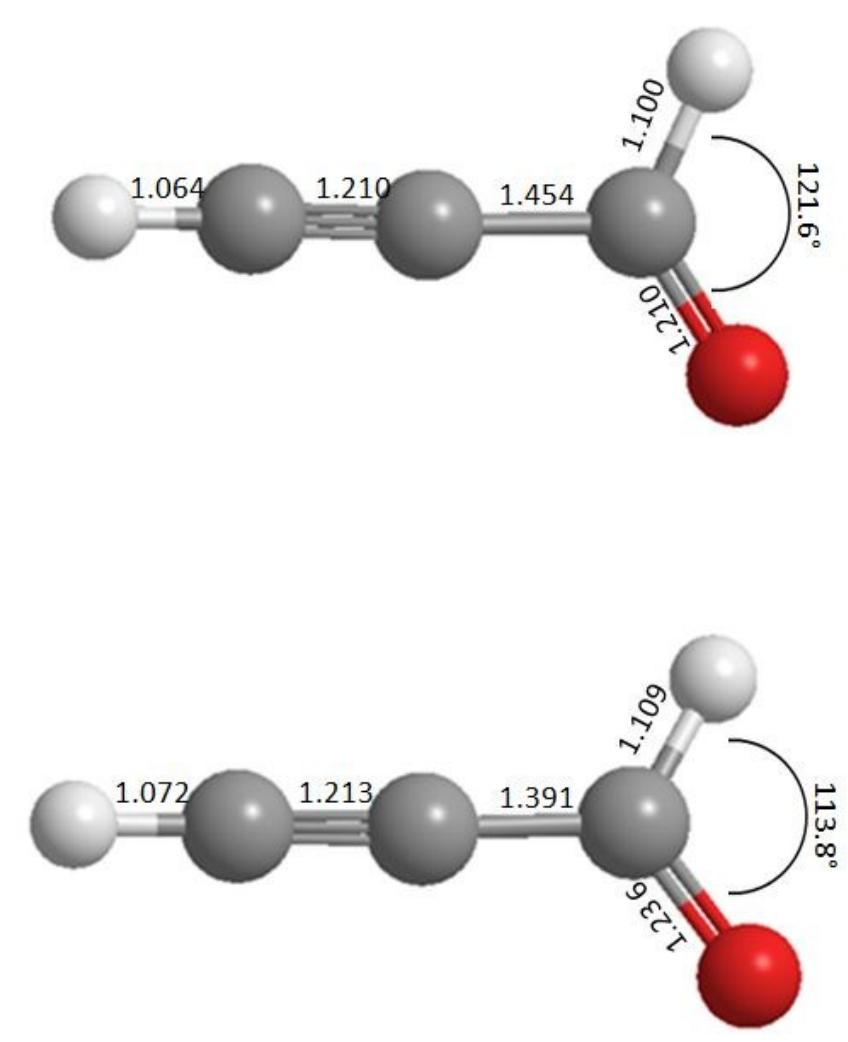

Figure 8: Optimized geometry of neutral propynal (upper panel) and its cation (lower panel), at the (R)CCSD(T)-F12 level using the the aug-cc-pVTZ basis set. 


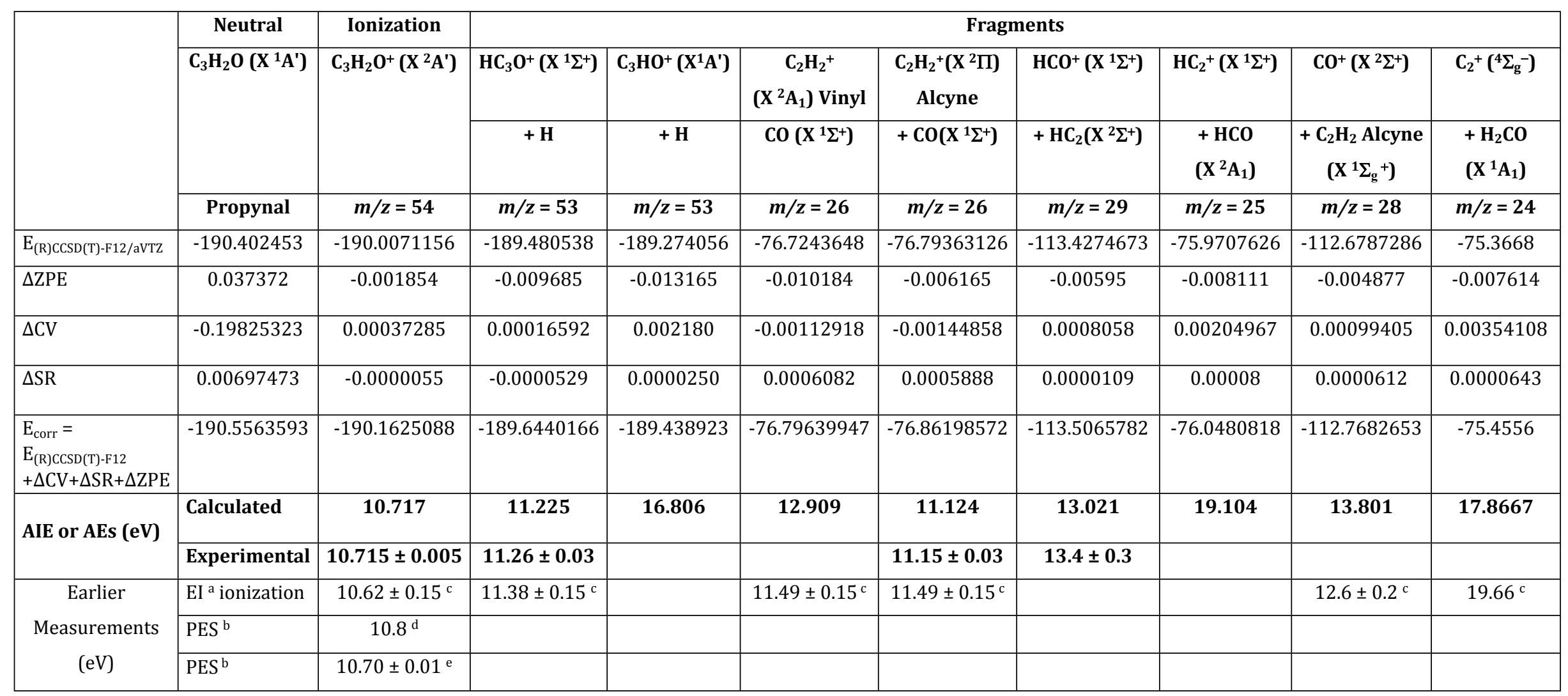

${ }^{\mathrm{a}} \mathrm{EI}=$ electron impact, ${ }^{\mathrm{b}} \mathrm{PES}=$ photoelectron spectroscopy, ${ }^{\mathrm{c}}$ ref. $14^{\mathrm{d}}$ ref. $40 ;{ }^{\mathrm{e}}$ ref. 10

Table 1: Calculated and measured adiabatic ionization energies (AIE) propynal and the appearance energies (AEs) of the different fragmentation pathways of the resulting cation (in bold, in eV). Earlier measurements are also given for comparison. Details on the calculations are also shown (energies in Hartree). $\triangle \mathrm{ZPE}$ is evaluated at the PBE0/aVTZ level using the corresponding anharmonic frequencies. The equilibrium structure of these species is given in Table $\mathrm{S} 1$ of the supplementary material. 

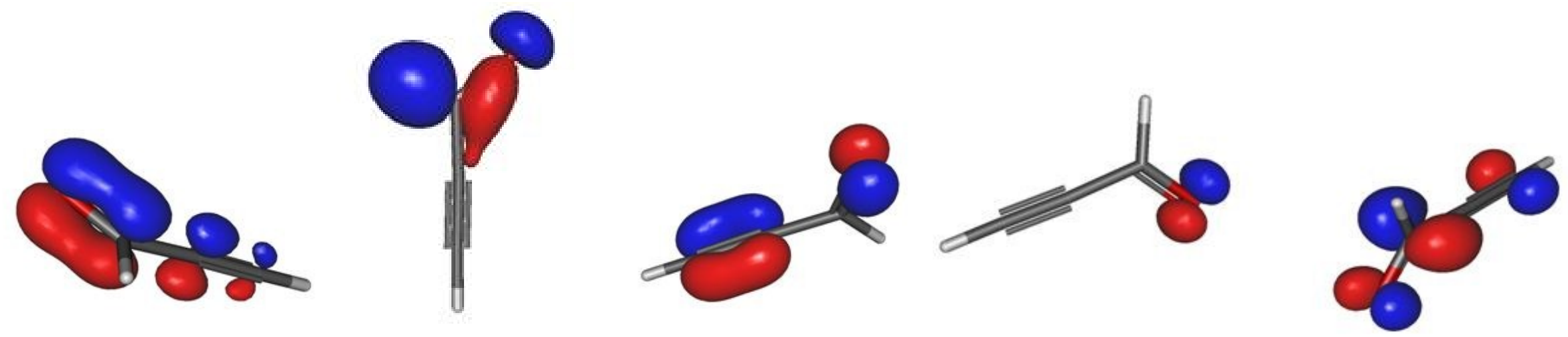

Table 2: Outermost Molecular Orbitals (MOs) of neutral propynal calculated using the (R)CCSD(T)-F12mthode with aug-cc-pVTZ basis set. 


\begin{tabular}{|c|c|c|c|}
\hline \multirow{3}{*}{ State } & \multirow{3}{*}{ Electron configuration } & \multicolumn{2}{|r|}{$\Delta \mathbf{E}$} \\
\hline & & Theory & Experiment \\
\hline & & MRCI & TPEPICO \\
\hline$\widetilde{X}^{2} \mathrm{~A}^{\prime}$ & $(0.81)\left\{1 a^{\prime \prime 2} 11 a^{\prime 2} 2 a^{\prime 2} 12 a^{\prime 1}\right\}$ & $0.00^{\text {a) }}$ & $0.00^{\text {a) }}$ \\
\hline $1^{2} \mathrm{~A}^{\prime \prime}$ & $(0.93)\left\{1 a^{\prime \prime 2} 11 a^{\prime 2} 2 a^{\prime \prime} 12 a^{\prime 2}\right\}$ & 0.65 & $0.69 \pm 0.02$ \\
\hline $2^{2} \mathrm{~A}^{\prime}$ & $(0.80)\left\{1 a^{\prime \prime 2} 11 a^{\prime 1} 2 a^{\prime 2} 12 a^{\prime 2}\right\}$ & 0.76 & $0.81 \pm 0.03$ \\
\hline $2^{2} \mathrm{~A}^{\prime \prime}$ & $(0.89)\left\{1 a^{\prime \prime} 11 a^{\prime 2} 2 a^{\prime 2} 12 a^{\prime 2}\right\}$ & 3.65 & $3.74 \pm 0.1$ \\
\hline $3^{2} \mathrm{~A}^{\prime \prime}$ & $(0.80)\left\{1 \mathrm{a}^{\prime 2} 11 \mathrm{a}^{\prime 1} 2 \mathrm{a}^{\prime 2} 12 \mathrm{a}^{\prime 1} 3 \mathrm{a}^{\prime 1}\right\}$ & 4.06 & \\
\hline $3^{2} \mathrm{~A}^{\prime}$ & $(0.67)\left\{1 a^{\prime \prime 2} 11 a^{\prime 1} 2 a^{\prime \prime} 12 a^{\prime 2} 3 a^{\prime 1}\right\}$ & 4.13 & $4.1 \pm 0.1$ \\
\hline $4^{2} \mathrm{~A}^{\prime \prime}$ & $(0.76)\left\{1 a^{\prime \prime 2} 11 a^{\prime 1} 2 a^{\prime 2} 12 a^{\prime 1} 3 a^{\prime 1}\right\}$ & 4.30 & \\
\hline $4^{2} \mathrm{~A}^{\prime}$ & $(0.58)\left\{1 a^{\prime \prime 2} 11 a^{\prime 2} 2 a^{\prime \prime} 12 a^{\prime 1} 3 a^{\prime \prime}\right\}$ & 5.82 & \\
\hline
\end{tabular}

a) Used as reference.

Table 3: Dominant electron configurations and vertical excitation energies $(\Delta E, e V)$ of the lowest doublet electronic states of propynal ${ }^{+}$ion. Between parentheses, we give the weight of this configuration in the corresponding wavefunction. 


\begin{tabular}{|c|c|c|c|}
\hline Mode & Symm & $\mathrm{E}_{\text {anharm }}$ & Tentative attribution \\
\hline$v_{1}$ & $\mathrm{a}^{\prime}$ & 3258 & $\mathrm{C}-\mathrm{H}$ stretch $(\mathrm{C} \equiv \mathrm{C}-\mathrm{H})$ \\
\hline$v_{2}$ & $\mathrm{a}^{\prime}$ & 2742 & $\mathrm{C}-\mathrm{H}$ stretch$(\mathrm{H}-\mathrm{C}=\mathrm{O})$ \\
\hline$v_{3}$ & $\mathrm{a}^{\prime}$ & 2179 & $\mathrm{C} \equiv \mathrm{C}$ stretch \\
\hline$v_{4}$ & $\mathrm{a}^{\prime}$ & 1527 & $\mathrm{C}=\mathrm{O}$ stretch \\
\hline$v_{5}$ & $\mathrm{a}^{\prime}$ & 1129 & $\mathrm{C}-\mathrm{H}$ rocking \\
\hline$v_{6}$ & $\mathrm{a}^{\prime}$ & 996 & $\mathrm{C}-\mathrm{H}$ wagging \\
\hline$v_{7}$ & $\mathrm{a}^{\prime}$ & 742 & $\mathrm{C} \equiv \mathrm{C}-\mathrm{H}$ in-plane bend \\
\hline$v_{8}$ & $\mathrm{a}^{\prime}$ & 418 & $\mathrm{CCO}$ in-plane bend \\
\hline$v_{9}$ & $\mathrm{a}^{\prime}$ & 144 & $\mathrm{CC} \equiv \mathrm{C}$ in-plane bend \\
\hline$v_{10}$ & $\mathrm{a}^{\prime \prime}$ & 875 & $\mathrm{C}(\mathrm{O}) \mathrm{H}$ out-of-plane bend \\
\hline$v_{11}$ & $\mathrm{a}^{\prime \prime}$ & 856 & $\mathrm{C} \equiv \mathrm{C}-\mathrm{H}$ out-of-plane bend \\
\hline$v_{12}$ & $\mathrm{a}^{\prime \prime}$ & 256 & $\mathrm{CC} \equiv \mathrm{C}$ out-of-plane bend \\
\hline
\end{tabular}

Table 4: Anharmonic wavenumbers $\left(\mathrm{E}_{\text {anharm }}, \mathrm{cm}^{-1}\right)$ of the fundamental bands of the $\mathrm{C}_{3} \mathrm{H}_{2} \mathrm{O}^{+}$ion in its electronic ground state as computed at the PBE0/aug-cc-pVDZ level of theory. 


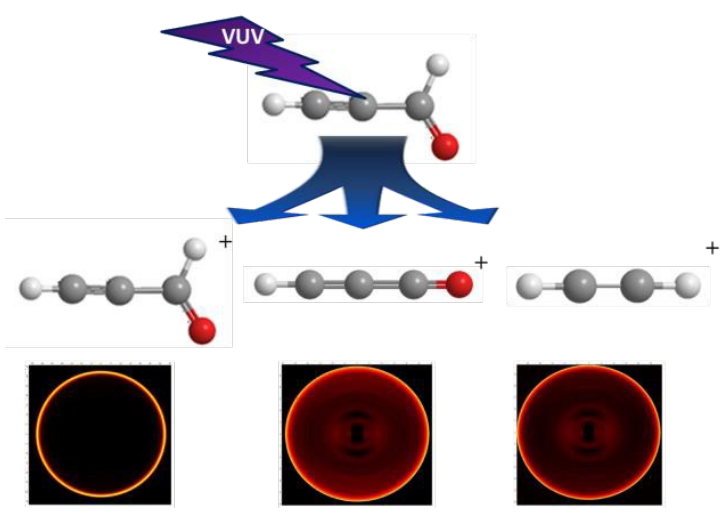

Figure for graphical abstract 